\title{
EDITORIAL
}

\section{Sarcoidosis from bench to bedside: a state-of-the-art series for the clinician}

\author{
Vincent Cottin*,\# and Joachim Müller-Quernheim $^{\star}$
}

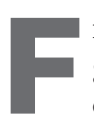

irst described in 1899 [1], sarcoidosis is a multi-organ granulomatous disorder that remains an enigma and challenges researchers and clinicians due to its unknown aetiology, variegated presentation and an unpredictable, and occasionally severe or even fatal, outcome despite therapy. As over $90 \%$ of patients have involvement of the lungs and thoracic lymph nodes, most chest physicians encounter sarcoidosis regularly in their practice, and have to manage this disorder with a risk of delayed or inadequate care [1].

Sarcoidosis may cause significant morbidity, as it persists as a chronic disease in approximately one-third of cases [1]. It most commonly affects individuals aged 20-39 yrs [2]. The prevalence of disease varies widely throughout the world and in ethnic groups, with a mean estimate of 15 cases per 100,000 persons, roughly corresponding to one in 6,000 persons in the general population [3]. In Ireland, England, central Europe and Scandinavia the prevalence ranges between 40 and 60 cases per 100,000 and associations with environmental and occupational exposure have been reported [4-8].

In the past, the European Respiratory Journal (ERJ) has disseminated advances in knowledge of sarcoidosis and is committed to doing so consistently. To this end, the ERJ has, in the June issue, started a new series on sarcoidosis that will provide an in-depth overview of the tremendous progress made in this field in recent years. This series of articles intends to provide readers with the most up-to-date and comprehensive reviews. The authors are among the world's leading experts in the field of sarcoidosis, and the articles are intended to be practically oriented and relevant for practicing physicians without neglecting the cutting edge of research. Topics developed in the series were carefully chosen as those with the most recent and important developments, be it in the setting of clinical manifestations, investigations, pathophysiology or therapeutic approach; some articles are related to approaches already applicable in practice, while others are anticipating developments in the near future.

One of the highlights of the series will consist of two articles on the imaging of sarcoidosis. The diagnosis of sarcoidosis is

\footnotetext{
*Hospices Civils de Lyon, Hôpital Louis Pradel, National Reference Centre for Rare Pulmonary Diseases, Competence centre for pulmonary hypertension, Dept of respiratory medicine and \#University of Lyon, University Claude Bernard Lyon I, INRA, UMR754, IFR 128, Lyon, France. 'Dept of Pneumology, University Medical Center, Freiburg, Germany.

CORRESPONDENCE: V. Cottin, Hôpital Louis Pradel, Service de Pneumologie, 28 Avenue Doyen Lepine, F-69677 Lyon, France. E-mail: vincent.cottin@chu-lyon.fr
}

based on compatible clinical and radiological manifestations, with histologic evidence of noncaseating epithelioid-cell granulomas in one or more organs and in the absence of identifiable cause, especially microorganism or exposure to antigens that may cause granulomas [9]. Therefore, chest imaging dominated by chest computed tomography (CT) is key to the diagnosis of sarcoidosis. T. Nunes will review the eminently variegated imaging features of sarcoidosis; the typical pattern which is highly suggestive of the diagnosis when predominant (i.e. bilateral hilar lymphadenopathy with peri-lymphatic micronodules), or bilateral hilar retractile masses with scarring and traction bronchiectasis (characteristic of stage IV sarcoidosis). Less characteristic patterns include cavitation, nodular and alveolar opacities, and the recently described patterns of sarcoid galaxy and sarcoid cluster, as well as rare patterns (ground-glass opacities, linear opacities and cystic destruction). The authors will further review the evidence regarding imaging and functional correlations at baseline (including various features associated with airflow limitation) and during evolution (imaging as predictor of outcome), and the impact of imaging evaluation on clinical management. Examples are provided of how chest CT can guide diagnostic interventions [10] and may allow the diagnosis of most complications that carry significant morbidity in pulmonary sarcoidosis.

${ }^{18} \mathrm{~F}$ fluorodeoxyglucose positron emission tomography (PET) scanning identifies areas with active metabolic (inflammatory) activity [11], which can be targeted by biopsies [12], and suggests the presence of disease in organs that are difficult to access and with potential morbidity, especially the brain and the heart. PET scanning has progressively replaced gallium-67 scanning in most centres. However, it is expensive, nonspecific and associated with significant radiation; therefore, it can't be routinely recommended in patients with sarcoidosis. In the article by J. Grutters, the authors synthesise the available evidence and suggest situations where PET scanning is useful, especially in the diagnosis of cardiac sarcoidosis, provided that images are acquired specifically to address this question and in close collaboration with knowledgeable specialists in nuclear imaging.

Among all organs (all of which can be involved by sarcoidosis), the heart is the one associated with the most difficult challenge in diagnosis and management. U. Costabel will review the accumulating evidence that cardiac sarcoidosis is more common than previously evaluated, although often not causing any clinical manifestation [13], and occasionally occurring in the absence of apparent disease elsewhere in the body. Potentially life-threatening, heart involvement by sarcoidosis may be found 
in up to $25 \%$ of patients during post mortem in the USA [14], and can affect any part of the heart especially the conducting system (causing complete heart block) and the myocardium (with granulomas and fibrosis causing heart failure, syncope or sudden death due to ventricular arrhythmias) [1]. The relative strengths and weaknesses of available investigations will be discussed, with the most commonly used tests being PET scanning (the results of which can be affected by antiinflammatory drugs) and delayed enhancement magnetic resonance imaging (which is not widely available). U. Costabel will address the question of whether cardiac sarcoidosis detected by systematic imaging techniques in asymptomatic patients requires treatment. Indications for electrocardiogram, echocardiography, cardiac electric monitoring and advance electrophysiologic studies will also be discussed, as well as involvement of other non-thoracic organs relevant for chest physicians.

Another issue of particular interest is that of chronic fatigue, a disabling symptom causing impaired quality of life and reported in up to $50-80 \%$ of sarcoidosis patients [15], as reviewed by DRENT et al. [16] in this issue of the ERJ. Usually multifactorial and enhanced by comorbidities (including anaemia, depression, anxiety, hypothyroidism, altered sleep patterns, etc.), and possibly by complications of corticosteroid therapy, fatigueassociated sarcoidosis may persist despite the treatment of possible causes, and is not correlated with clinical parameters of disease activity. This suggests that other pathogenic factors may take place, providing targets for specific therapy once identified.

Two articles in the series are devoted to the therapy and management of patients with sarcoidosis, with a focus on severe complications of the disease. R. Baughman will discuss the standard treatment for pulmonary sarcoidosis, especially corticosteroids (which represent the standard therapy) and steroid-sparing agents such as methotrexate, azathioprine and leflunomide [17]. Important practical considerations concern the indications for therapy and measures used to assess the response. The authors will further review the potential role of newer biological agents (infliximab and adalimumab) that are being evaluated in patients with sarcoidosis especially those with extra-pulmonary refractory involvement, although the role of these agents in the management of patients with pulmonary sarcoidosis is currently marginal [18].

Although a minority of sarcoidosis patients progress to advanced stages of their disease, complications at this stage are challenging. In a recent issue of the ERJ, SCHLOBIN and NATHAN [19] comprehensively reviewed the evidence regarding pulmonary hypertension associated with sarcoidosis. Seven case series and one clinical trial [20-27] using therapy specific for pulmonary arterial hypertension collectively suggest a possible benefit of these agents in specific patients with sarcoidosis-associated pulmonary hypertension, although this requires further study. SCHLOBIN and NATHAN [19] also discussed the specificities of lung transplantation in the setting of sarcoidosis, with the main particularities being the multi-organ involvement, the risk of infection (fungal infections developing in cavities) and surgical difficulties due to bulky hilar adenopathy and perihilar fibrosis, pleural thickening or pulmonary hypertension.

More fundamental issues are addressed in the final three articles of the series, namely genetics, immunopathogenesis and biomarker development; topics that are immediately relevant for clinicians. Familial clustering of sarcoidosis may be found in $5-10 \%$ of patients, and represents, together with genome-wide scanning for susceptibility genes [28], one of the best ways to progress in the understanding of this disease of elusive aetiology. In 2005, dentification of butyrophilin-like-2 as a main genetic determinant of sarcoidosis was reported [29]. This was a landmark discovery that has stimulated further research in sarcoidosis genetics, yielding new susceptibility gene variants $[28,30]$ and even susceptibility loci shared by sarcoidosis and another granulomatous disorder [31]. Recent progress in the immunopathogenesis of this condition notably includes the probable role of regulatory $\mathrm{T}$-cell lymphocytes and natural killer T-cells in granuloma formation, and the role of the shift from T-helper 1 to T-helper 2 lymphocytes in fibrogenesis. Finally, P. Rottoli will review the state-of-the-art regarding biomarker development in sarcoidosis and the search for simple tests that are useful for diagnosis and assessing disease activity.

We hope readers of the ERJ will enjoy reading these outstanding series articles, which will enhance our clinical skills and our comprehension of disease pathogenesis.

\section{STATEMENT OF INTEREST}

A statement of interest for V. Cottin can be found at www.erj. ersjournals.com/site/misc/statements.xhtml

\section{REFERENCES}

1 Iannuzzi MC, Fontana JR. Sarcoidosis: clinical presentation, immunopathogenesis, and therapeutics. JAMA 2011; 305: 391-399.

2 Rybicki BA, Major M, Popovich J Jr, et al. Racial differences in sarcoidosis incidence: a 5-year study in a health maintenance organization. Am J Epidemiol 1997; 145: 234-241.

3 Orphanet Report Series. Prevalence of rare disease: bibliographic data. Number 1. November 2011. www.orpha.net/orphacom/ cahiers/docs/GB/Prevalence_of_rare_diseases_by_alphabetical_ list.pdf

4 Gorham ED, Garland CF, Garland FC, et al. Trends and occupational associations in incidence of hospitalized pulmonary sarcoidosis and other lung diseases in navy personnel: a 27-year historical prospective study, 1975-2001. Chest 2004; 126: 1431-1438.

5 Kreider ME, Christie JD, Thompson B, et al. Relationship of environmental exposures to the clinical phenotype of sarcoidosis. Chest 2005; 128: 207-215.

6 Drent M, Wijnen PA, Boots AW, et al. Cat litter is a possible trigger for sarcoidosis. Eur Respir J 2012; 39: 221-222.

7 Rafnsson V, Ingimarsson $\mathrm{O}$, Hjalmarsson I, et al. Association between exposure to crystalline silica and risk of sarcoidosis. Occup Environ Med 1998; 55: 657-660.

8 Baughman RP, Teirstein AS, Judson MA, et al. Clinical characteristics of patients in a case control study of sarcoidosis. Am J Respir Crit Care Med 2001; 164: 1885-1889.

9 Statement on sarcoidosis. Joint statement of the American Thoracic Society (ATS), the European Respiratory Society (ERS) and the World Association of Sarcoidosis and Other Granulomatous Disorders (WASOG) adopted by the ATS board of directors and by the ERS Executive Committee, February 1999. Am J Respir Crit Care Med 1999; 160: 736-755.

10 Herth FJ, Schuler H, Gompelmann D, et al. Endobronchial ultrasound-guided lymph node biopsy with transbronchial needle forceps: a pilot study. Eur Respir J 2012; 39: 373-377. 
11 Braun JJ, Kessler R, Constantinesco A, et al. 18F-FDG PET/CT in sarcoidosis management: review and report of 20 cases. Eur J Nucl Med Mol Imaging 2008; 35: 1537-1543.

12 Teirstein AS, Machac J, Almeida O, et al. Results of 188 wholebody fluorodeoxyglucose positron emission tomography scans in 137 patients with sarcoidosis. Chest 2007; 132: 1949-1953.

13 Silverman KJ, Hutchins GM, Bulkley BH. Cardiac sarcoid: a clinicopathologic study of 84 unselected patients with systemic sarcoidosis. Circulation 1978; 58: 1204-1211.

14 Kim JS, Judson MA, Donnino R, et al. Cardiac sarcoidosis. Am Heart J 2009; 157: 9-21.

15 Marcellis RG, Lenssen AF, Elfferich MD, et al. Exercise capacity, muscle strength and fatigue in sarcoidosis. Eur Respir J 2011; 38: 628-634.

16 Drent M, Lower EE, De Vries J. Sarcoidosis-associated fatigue. Eur Respir J 2012; 40: 255-263.

17 Sahoo DH, Bandyopadhyay D, Xu M, et al. Effectiveness and safety of leflunomide for pulmonary and extrapulmonary sarcoidosis. Eur Respir J 2011; 38: 1145-1150.

18 Cottin V. Update on bioagent therapy in sarcoidosis. F1000 Med Rep 2010; 2: 13.

19 Shlobin OA, Nathan SD. Management of end-stage sarcoidosis: pulmonary hypertension and lung transplantation. Eur Respir J 2012; 39: 1521-1534.

20 Preston IR, Klinger JR, Landzberg MJ, et al. Vasoresponsivness of sarcoid-associated pulmonary hypertension. Chest 2001; 120: 866-872.

21 Baughman RP, Judson MA, Lower EE, et al. Inhaled iloprost for sarcoidosis associated pulmonary hypertension. Sarcoidosis Vasc Diffuse Lung Dis 2009; 26: 110-120.
22 Milman N, Burton CM, Iversen M, et al. Pulmonary hypertension in end-stage pulmonary sarcoidosis: therapeutic effect of sildenafil? J Heart Lung Transplant 2008; 75: 329-334.

23 Fisher KA, Serlin DM, Wilson KC, et al. Sarcoidosis-associated pulmonary hypertension: outcome with long-term epoprostenol treatment. Chest 2006; 130: 1481-1488.

24 Barnett CF, Bonura EJ, Nathan SD, et al. Treatment of sarcoidosisassociated pulmonary hypertension. A two-center experience. Chest 2009; 135: 1455-1461.

25 Culver DA, Minai OA, Chapman JT, et al. Treatment of pulmonary hypertension in sarcoidosis. Proc Am Thoracic Soc 2005; 2: A862.

26 Baughman RP, Engel PJ, Taylor L, et al. Survival in sarcoidosis associated pulmonary hypertension: the importance of hemodynamic evaluation. Chest 2010; 138: 1078-1085.

27 Judson MA, Ford HJ, Donohue JF, et al. Ambrisentan for sarcoidosis associated pulmonary hypertension. Am J Respir Crit Care 2010; 181: A2368.

28 Hofmann S, Fischer A, Till A, et al. A genome-wide association study reveals evidence of association with sarcoidosis at $6 \mathrm{p} 12.1$. Eur Respir J 2011; 38: 1127-1135.

29 Valentonyte R, Hampe J, Huse K, et al. Sarcoidosis is associated with a truncating splice site mutation in BTNL2. Nat Genet 2005; 37: 357-364.

30 Pabst S, Franken T, Schonau J, et al. Transforming growth factor- $\beta$ gene polymorphisms in different phenotypes of sarcoidosis. Eur Respir J 2011; 38: 169-175.

31 Fischer A, Nothnagel M, Franke A, et al. Association of inflammatory bowel disease risk loci with sarcoidosis, and its acute and chronic subphenotypes. Eur Respir J 2011; 37: 610-616. 